\title{
Relevance of topographic control on hydrogeological properties of the weathered granite gneiss aquifer in watershed perspective - Usri sub-basin, Giridih, India
}

\author{
Ashok Kumar ${ }^{1}$ \\ ${ }^{1}$ Vikramshila Society for Social \& Education Development
}

December 22, 2020

\begin{abstract}
Usri, a southward sloping fourth order drainage sub-basin of Barakar river, is located in the northern margin of Chhotanagpur plateau, Giridih, India. Gently sloping undulating landforms devoid of hills and outcrops is main characteristics of this subbasin. Topographic elevation varies between 310 to $390 \mathrm{~m}$ MSL. Geographically it is located between lat $24.38^{\circ} \mathrm{N}$ to $24.54^{\circ} \mathrm{N}$ long and $86.07^{\circ} \mathrm{E}$ and $86.28^{\circ} \mathrm{E}$ longitudes. Thick weathered horizon developed over homogenous Archean granite-gneiss is the principal unconfined aquifer system. Basement topographic (weathering depth) and water table have been correlated with the surface topographic features (landforms). Presence of considerably thick weathered horizons, devoid of rock exposures, regional uniformity in geomorphic characters and its location at fringe of plateau provides favorable condition for correlation of surface topography with basement topography and water table. It has been observed that basement surface is replica of ground surface only in regional or watershed perspective with reference to common datum. The basement surface is not always exact replica of ground surface at micro watershed scale. In many cases, basement surface is reverse of ground surface. The depth basement has remained constant along the basin water-divide situated near the margin of the plateau. The depth of basement (weathered horizon) is higher in upper reaches than lower reaches on the micro water-divides (upland) as well as in the drainage depressions (channels) within the sub-basin. The upper reaches of the sub-basin mainly along the basin water-divide has better groundwater prospects than lower reaches. There is no definite trend of water table with respect to ground and basement topography. Many places water table is shallow on the micro water-divides (upland) and deep in drainage depressions (channels). The established correlations are likely to be applicable in the other geographical area where similar watershed and geological characteristics exits.
\end{abstract}

\section{Hosted file}

Usri_Landform_Paper_without_Figures_Table.pdf available at https://authorea.com/users/384394/ articles/500014-relevance-of-topographic-control-on-hydrogeological-properties-of-theweathered-granite-gneiss-aquifer-in-watershed-perspective-usri-sub-basin-giridih-india

\section{Hosted file}

TABLE 1.pdf available at https://authorea.com/users/384394/articles/500014-relevanceof-topographic-control-on-hydrogeological-properties-of-the-weathered-granite-gneissaquifer-in-watershed-perspective-usri-sub-basin-giridih-india

\section{Hosted file}

TABLE 2\selectlanguage\{ngerman\} .pdf available at https://authorea.com/users/384394/articles/ 500014-relevance-of-topographic-control-on-hydrogeological-properties-of-the-weatheredgranite-gneiss-aquifer-in-watershed-perspective-usri-sub-basin-giridih-india 


\section{Hosted file}

TABLE 3.pdf available at https://authorea.com/users/384394/articles/500014-relevanceof-topographic-control-on-hydrogeological-properties-of-the-weathered-granite-gneissaquifer-in-watershed-perspective-usri-sub-basin-giridih-india

\section{Hosted file}

TABLE 4.pdf available at https://authorea.com/users/384394/articles/500014-relevanceof-topographic-control-on-hydrogeological-properties-of-the-weathered-granite-gneissaquifer-in-watershed-perspective-usri-sub-basin-giridih-india

\section{Hosted file}

TABLE 5.pdf available at https://authorea.com/users/384394/articles/500014-relevanceof-topographic-control-on-hydrogeological-properties-of-the-weathered-granite-gneissaquifer-in-watershed-perspective-usri-sub-basin-giridih-india

\section{Hosted file}

TABLE 6.pdf available at https://authorea.com/users/384394/articles/500014-relevanceof-topographic-control-on-hydrogeological-properties-of-the-weathered-granite-gneissaquifer-in-watershed-perspective-usri-sub-basin-giridih-india

\section{Hosted file}

TABLE 7.pdf available at https://authorea.com/users/384394/articles/500014-relevanceof-topographic-control-on-hydrogeological-properties-of-the-weathered-granite-gneissaquifer-in-watershed-perspective-usri-sub-basin-giridih-india

\section{Hosted file}

TABLE 8.pdf available at https://authorea.com/users/384394/articles/500014-relevanceof-topographic-control-on-hydrogeological-properties-of-the-weathered-granite-gneissaquifer-in-watershed-perspective-usri-sub-basin-giridih-india

\section{Hosted file}

TABLE 9.pdf available at https://authorea.com/users/384394/articles/500014-relevanceof-topographic-control-on-hydrogeological-properties-of-the-weathered-granite-gneissaquifer-in-watershed-perspective-usri-sub-basin-giridih-india

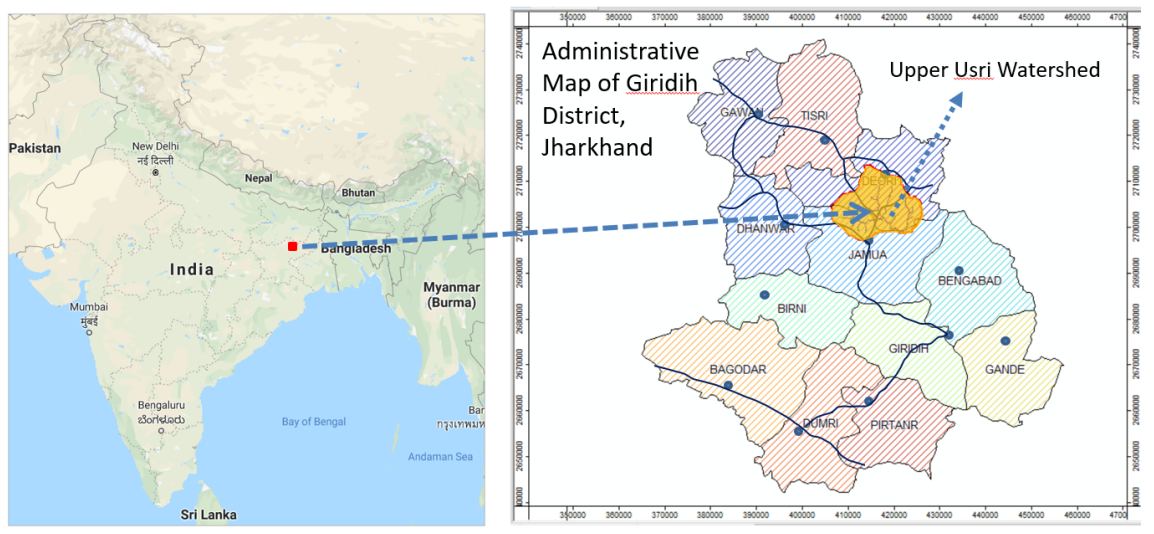



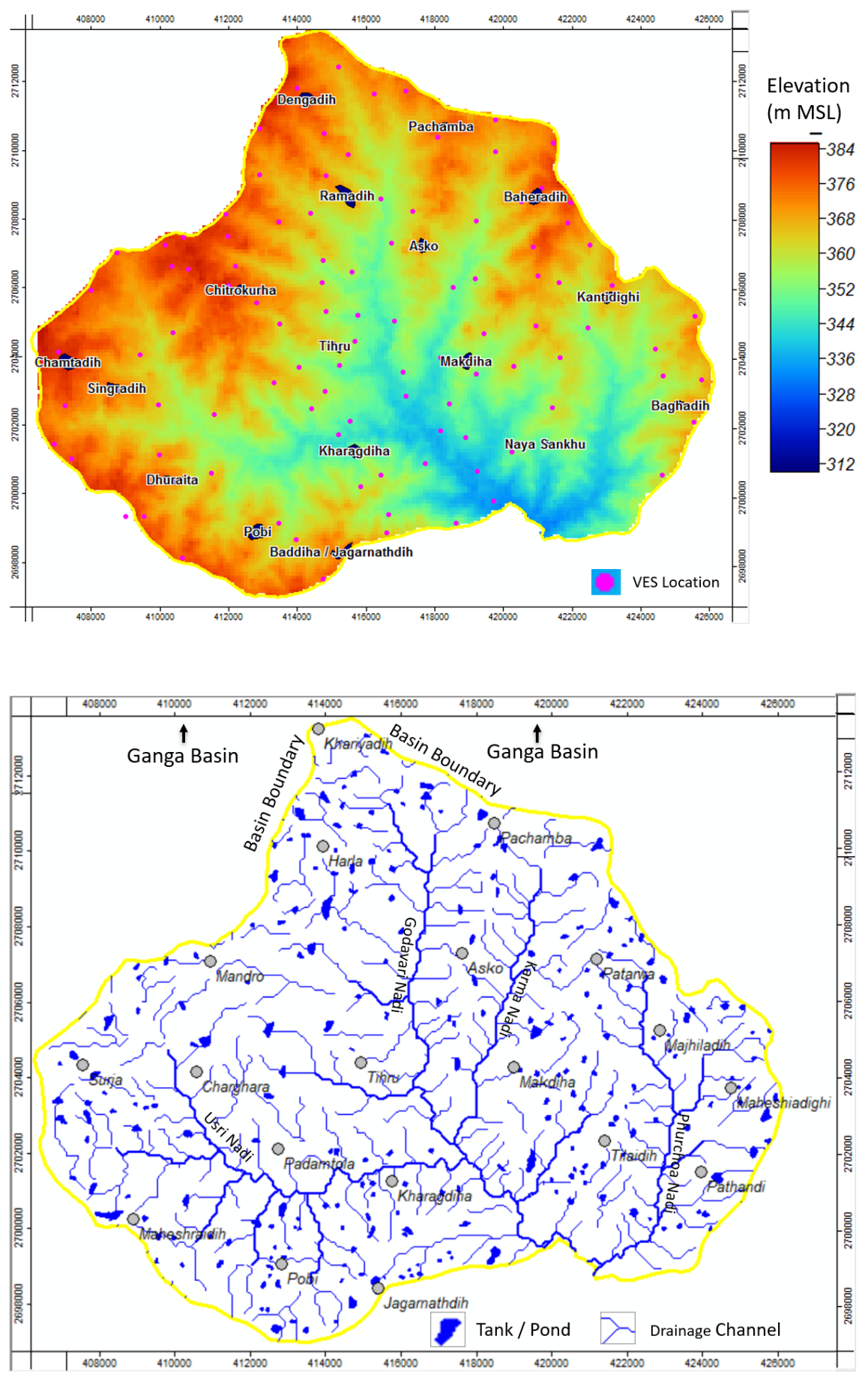

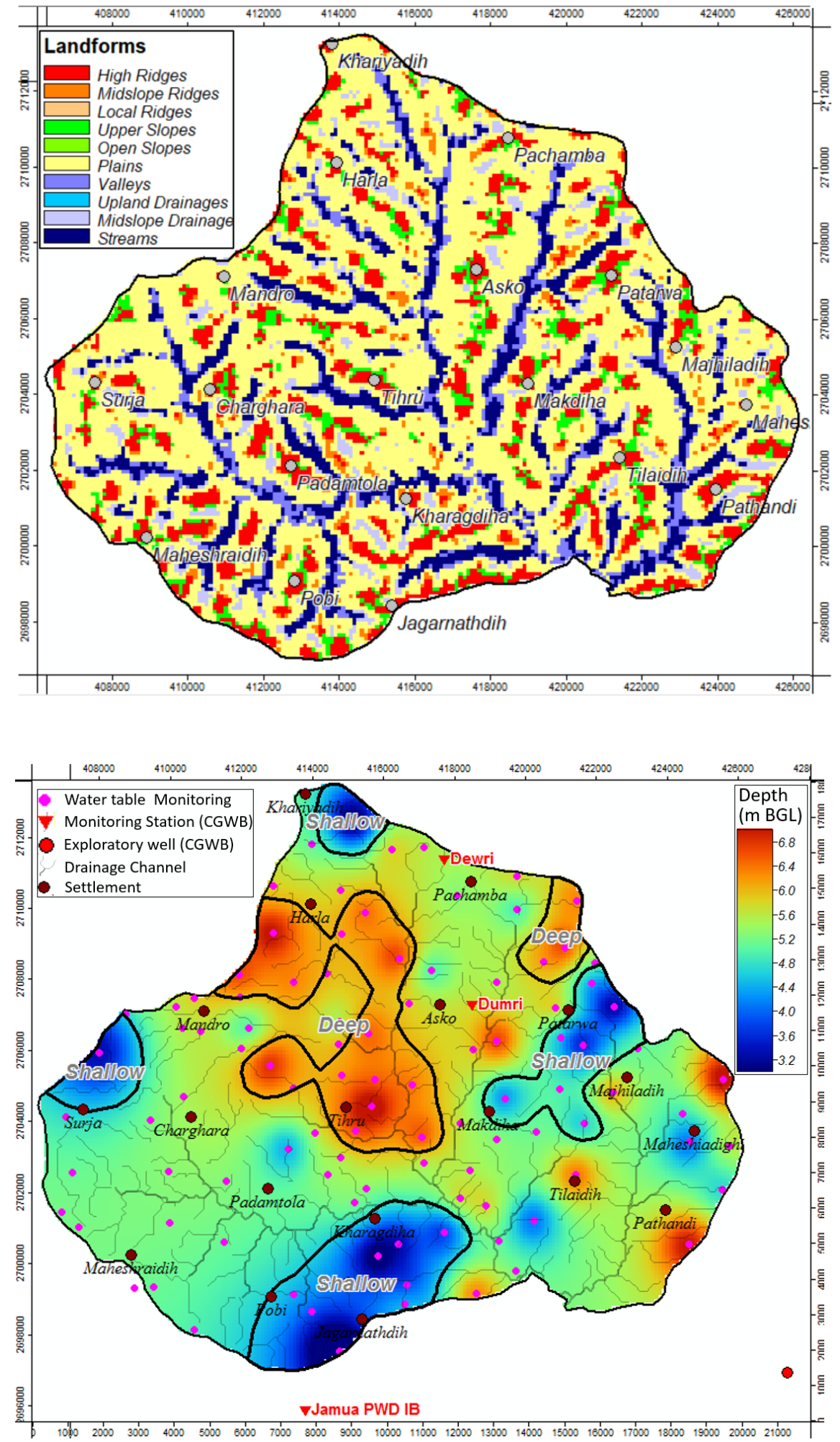

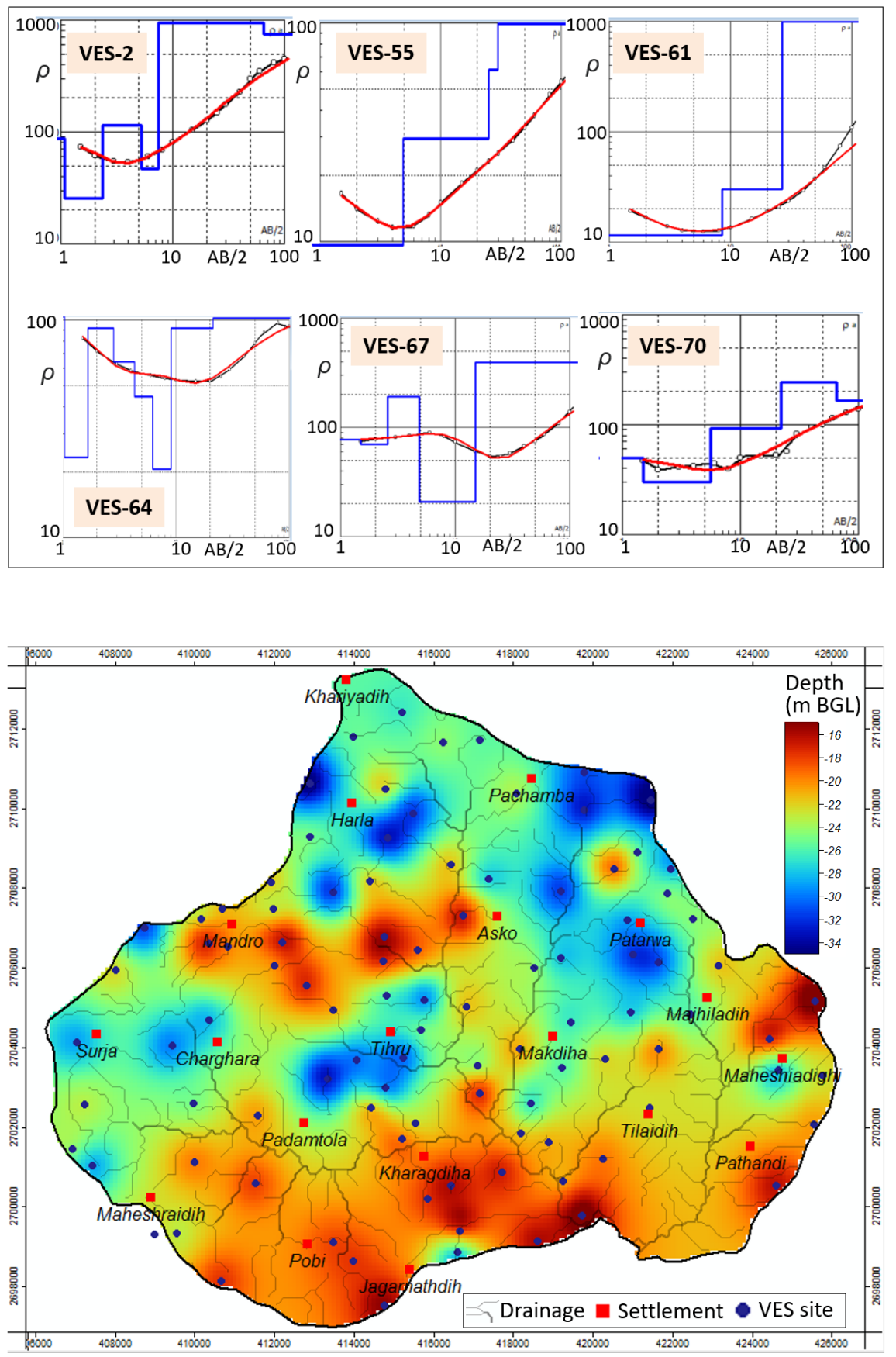

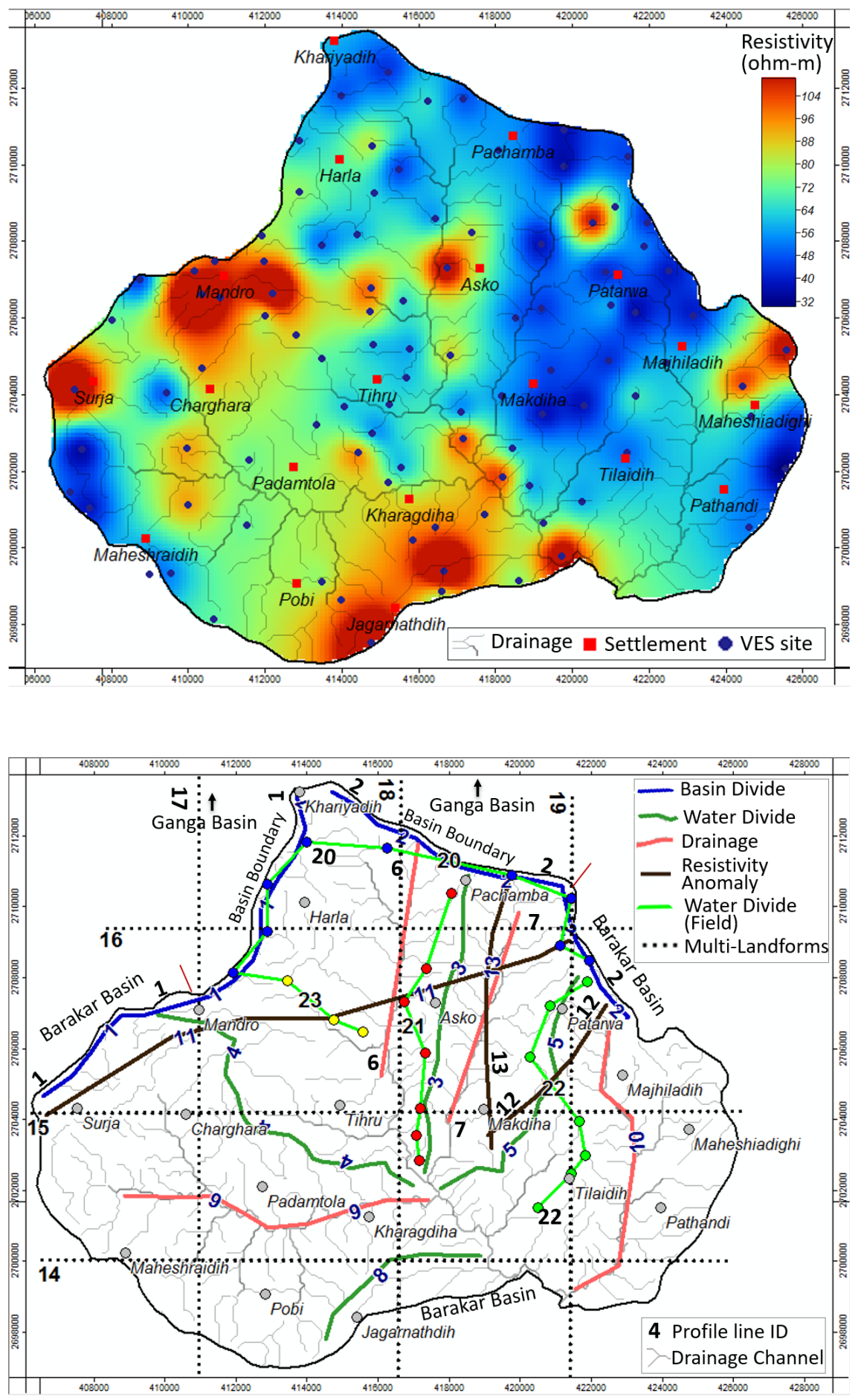

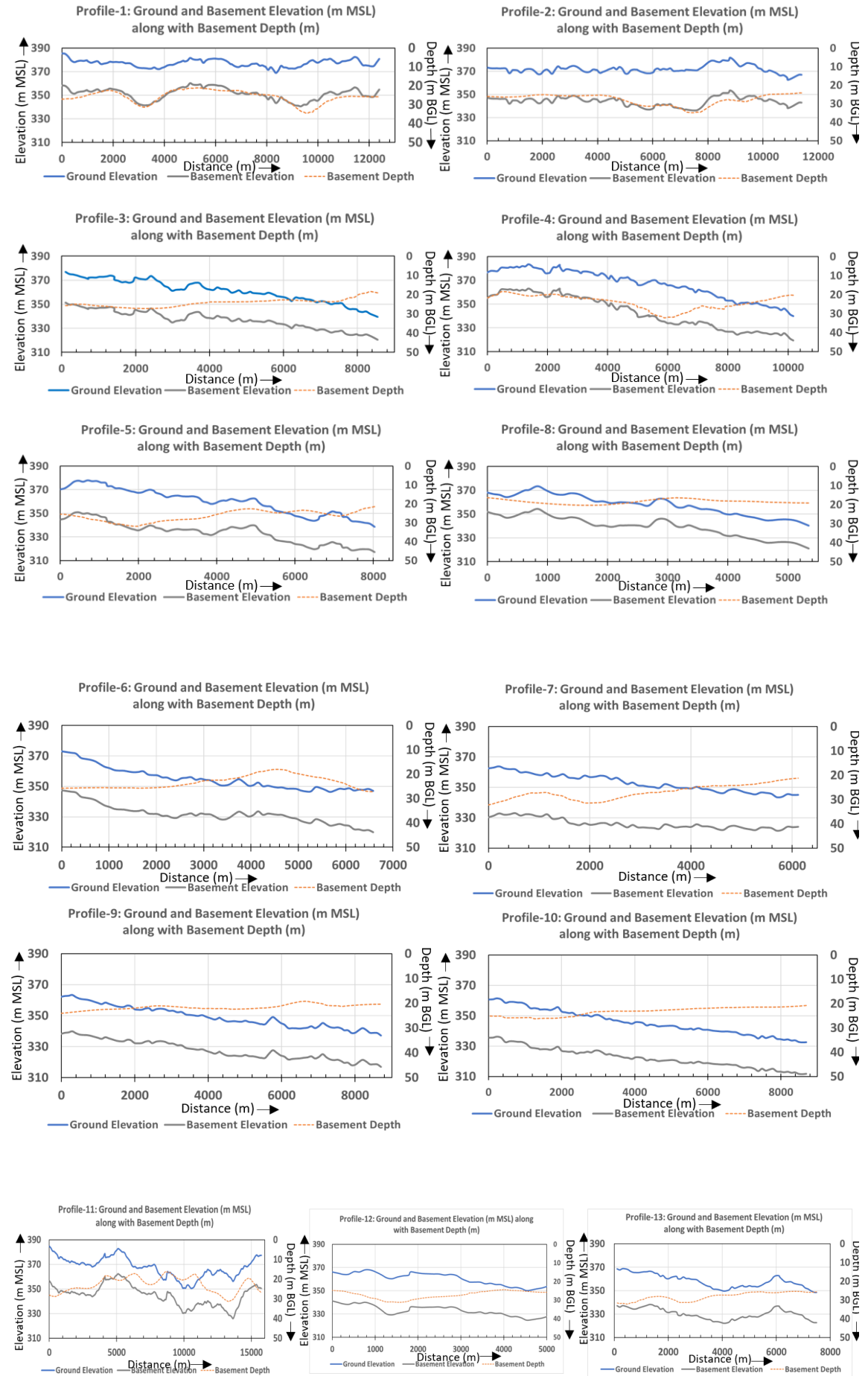

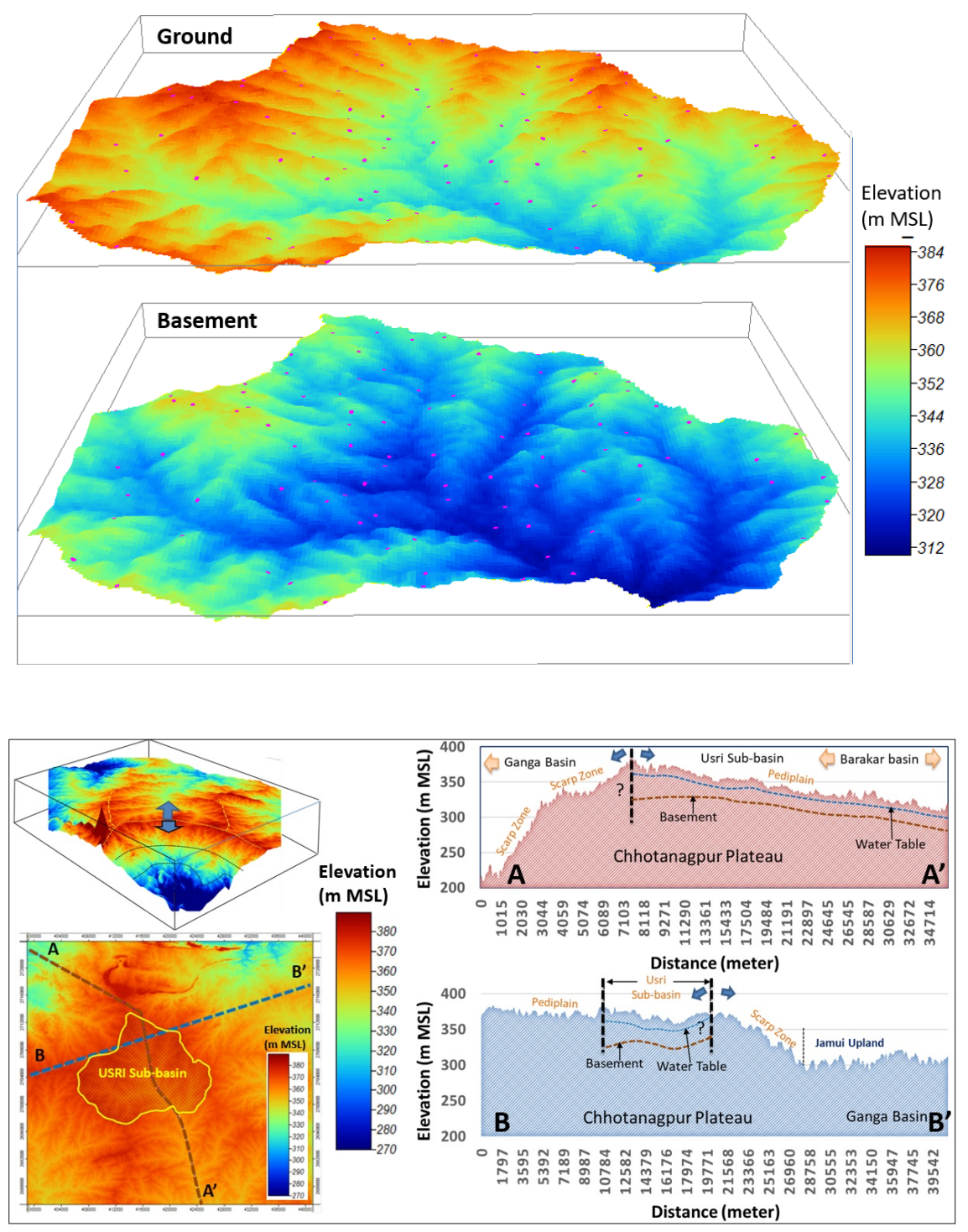
Line-1: Profile of Ground Elevation \& Water Table Depth

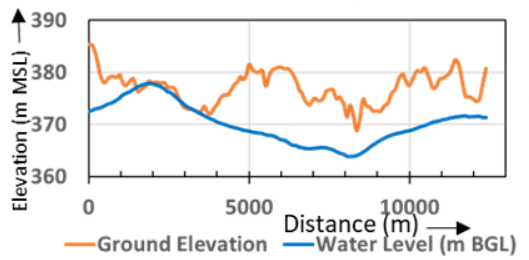

Line-3: Profile of Ground Elevation \& Water Table Depth

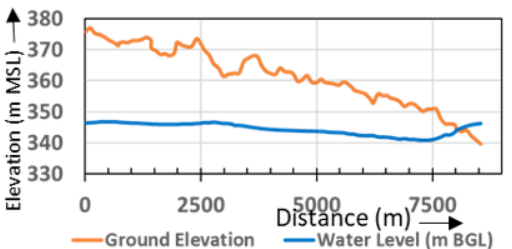

Line-5: Profile of Ground Elevation \& Water Table Depth

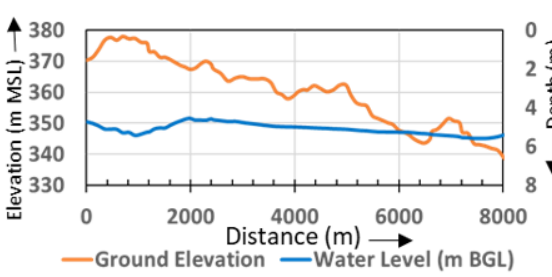

Water Divide Profile Line - 20 ( $\mathrm{T}$ - T') Profile running along northern extreme at basin divide)

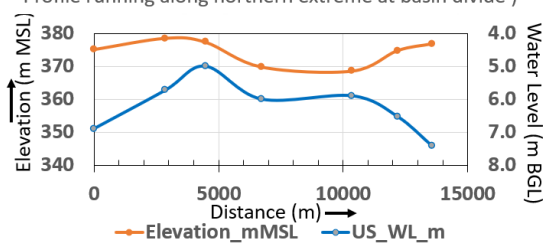

$\rightarrow$ Elevation_mMSL $\rightarrow$-US_WL_m

Water Divide Profile Line $-22\left(v-V^{\prime}\right)$

(NE - SW running profile line in eastern part sub-basin)

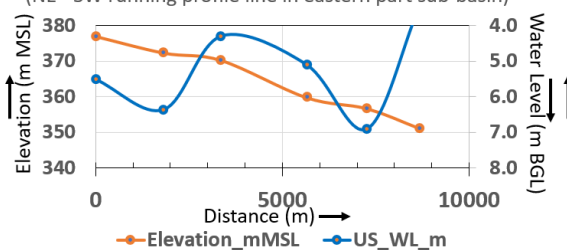

$\rightarrow-$ Elevation_mMSL $\rightarrow-$ US_WL_m
Line-2: Profile of Ground Elevation \& Water Table Depth

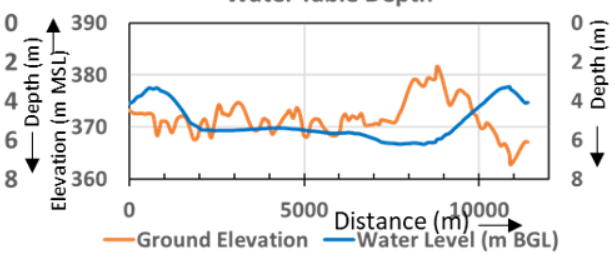

Line-4: Profile of Ground Elevation \& Water Table Depth

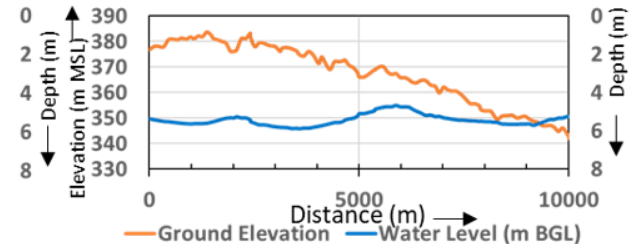

Line-8: Profile of Ground Elevation \& Water Table Depth

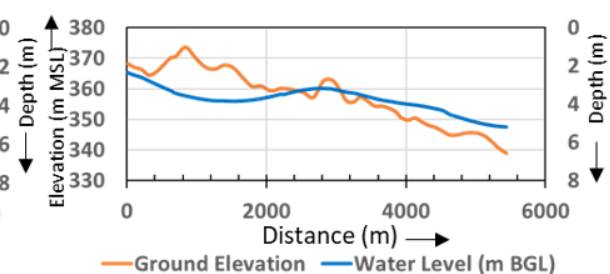

Water Divide Profile Line - $21\left(U-U^{\prime}\right)$

(NS running profile line in central part sub-basin)

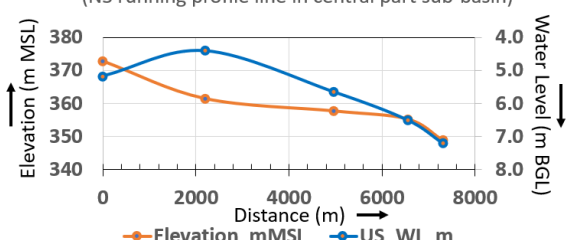

$\rightarrow$-Elevation_mMSL $\rightarrow$ - US_WL_m

Water Divide Profile Line - 23 (W- W') (NW-SE running profile line in NW area of sub-basin)

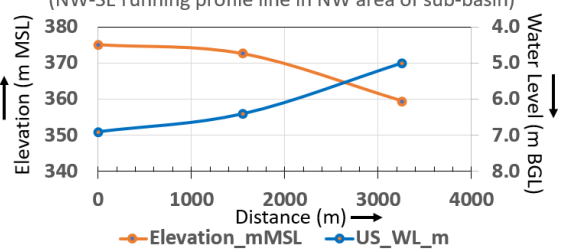




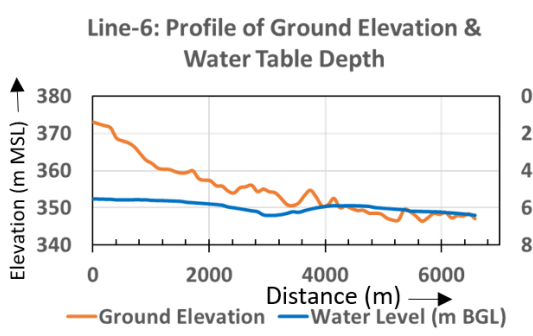

Line-7: Profile of Ground Elevation \& Water Table Depth
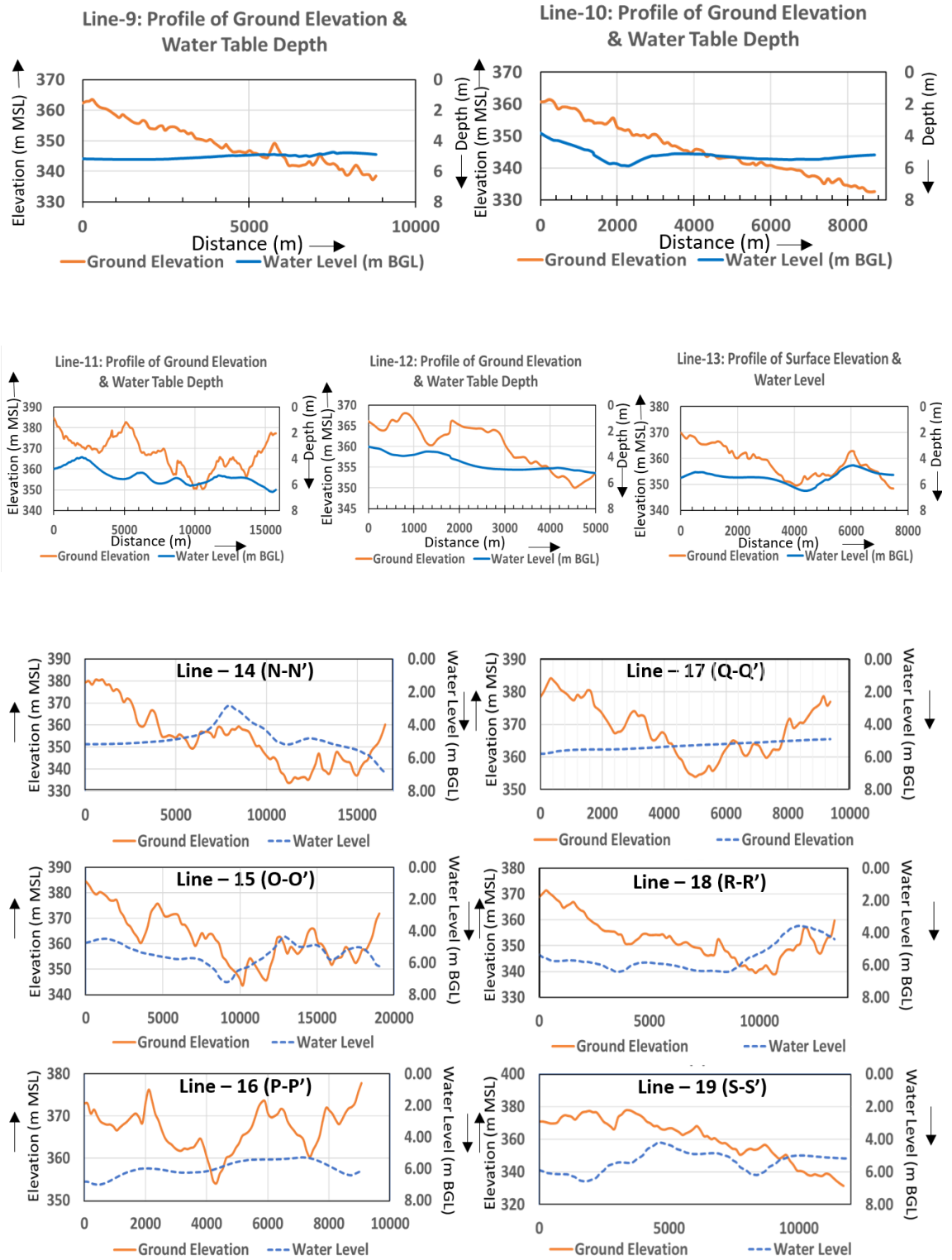